ECCOMAS

Proceedia
COMPDYN 2021

$8^{\text {th }}$ ECCOMAS Thematic Conference on Computational Methods in Structural Dynamics and Earthquake Engineering M. Papadrakakis, M. Fragiadakis (eds.)

\title{
MODELING AND DESIGN OF PERIODIC LATTICES WITH TENSEGRITY ARCHITECTURE AND HIGHLY NONLINEAR RESPONSE
}

\author{
Andrea Micheletti ${ }^{1}$, Claudio Intrigila ${ }^{1}$, Nicola Nodargi ${ }^{1}$, Edoardo Artioli ${ }^{1}$, Fernando \\ Fraternali $^{2}$, and Paolo Bisegna ${ }^{1}$ \\ ${ }^{1}$ Dipartimento di Ingegneria Civile e Ingegneria Informatica, University of Rome Tor Vergata \\ Via Politecnico 1, 00133, Rome, Italy \\ e-mail: micheletti, intrigila, nodargi, artioli@ing.uniroma2.it, bisegna@uniroma2.it \\ ${ }^{2}$ Department of Civil Engineering, University of Salerno, Italy \\ 84084 Fisciano (SA), Italy \\ e-mail: f.fraternali@unisa.it
}

\begin{abstract}
In recent years, the nonlinear response of tensegrity systems has attracted increasing attention in the study of mechanical metamaterials. It has been shown in the literature that geometry and prestress of an elastic tensegrity structure can be designed to obtain different behaviors: stiffening, softening, and snap-through behavior in statics; propagation of solitary waves in dynamics. However, the realization of tensegrity systems is challenging, because of their prestressed state and the presence of tension-only cable members. A design method for periodic lattices with null prestress and no cables is here proposed, in which the repeating unit is at, or close to, a tensegrity configuration, maintaining the nonlinear types of response aforementioned. These structures can be realized by conventional additive manufacturing techniques, while the static and dynamic response can be predicted by means of stick-and-spring models.
\end{abstract}

Keywords: Bistable Systems, Nonlinear Systems, Additive Manufacturing, Experimental Testing, Solitary Waves.

ISSN:2623-3347 C 2021 The Authors. Published by Eccomas Proceedia.

Peer-review under responsibility of the organizing committee of COMPDYN 2021.

doi: $10.7712 / 120121.8605 .19232$ 


\section{INTRODUCTION}

Nonlinear mechanical metamaterials have been the subject of numerous studies aimed at discovering and exploiting new structural architectures with unprecedented properties [1-3]. Among lattice metamaterials, tensegrity periodic lattices have been found to possess a peculiar types of behavior, owing to the highly nonlinear mechanical response of the constituting tensegrity units, and to the fact that such response can be tuned by changing their geometry or prestress level [4-12]. In particular, in [4,5], the nonlinear static and dynamic response of the triangular tensegrity prims (T3) was examined. In [6,7], it was shown that a T3 can feature a stiffening or a softening response to applied loads in dependence of its aspect ratio. In [8], two distinct regimes of bistability have been highlighted in a case study. In $[9,11]$, the impact dynamics of tensegrity columns was investigated, finding that such periodic structures support the propagation of solitary waves. In addition, it was shown in [10] that such solitary waves can be either compression or rarefaction waves according to the geometry of the repeating unit. In [12], compression compact waves were obtained in numerical simulations of tensegrity beams in twoand three-dimensions.

While tensegrity units have a rich collection of mechanical responses which are attractive for applications, prototyping and realization of tensegrity metamaterials are challenging, since they are largely composed of tension-only prestressed cables [13]. Taking the cue from the study reported in [14], where bistable lattices with tensegrity architecture were designed, fabricated at the microscale using multiphoton lithography, and subsequently subjected to loading-unloading compression testing, in this work we aimed at showing that similar structures can be realized at higher scales with relatively inexpensive additive manufacturing techniques, such as fused deposition modeling (FDM), in order to obtain lattice metamaterials with customizable nonlinear response, possessing no cables and no prestress. We considered one of the systems analyzed in [14], a double-T3 structure, and we fabricated it by FDM after redesigning its geometry. More specifically, the system was conceived as a compliant mechanism by realizing nodal member connections as flexible hinges $[15,16]$. The fabricated structure was then subjected to compression testing for determining its static response. The results of this test were used to validate a Stick-and-Spring reduced-order model of the structure $[17,18]$ and its predictions. The validated model was then used to perform impact dynamics simulations on lattice columns composed of T3 units.

This paper is organized as follows. After outlining the basic properties of tensegrity systems which can be inherited by tensegrity-like lattices in Section 2, the additive manufacturing process and compression testing results are described in Section 3. Afterward, the reduced-order Stick-and-Spring model, and the results of corresponding static and dynamic simulations are presented in Section 4, while our concluding remarks follow in Section 5.

\section{NONLINEAR LATTICES WITH TENSEGRITY ARCHITECTURE}

Tensegrity structures are prestressed pin-connected frameworks composed of cables and bars, which in many instances possess internal mechanisms stabilized by their prestress state. In these cases, the mechanical response is mainly geometrical, that is, it is associated to large configuration changes. The two-bar system depicted in Fig. 1(a) exemplifies this situation. In the reference configuration (in black in Fig. 1(a)), the two bars are aligned and subjected to a tensile prestressing axial force. The relation between the vertical load $F$ and the corresponding displacement $\delta$ is well approximated by a cubic whose slope in the origin, $\tan \alpha$, is directly proportional to the prestressing axial force, while a stiffening effect can be observed when $F$ 
increases.
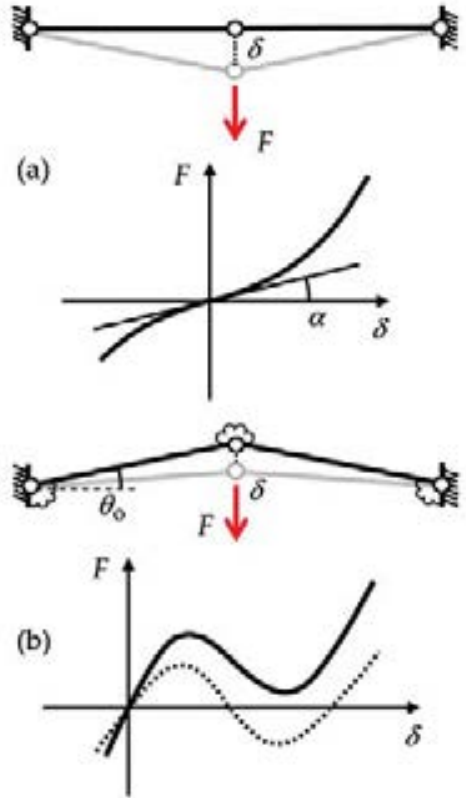

(c)



(d)

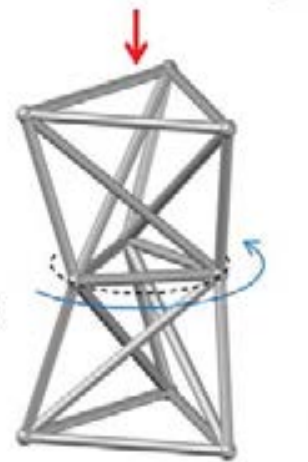

(e)

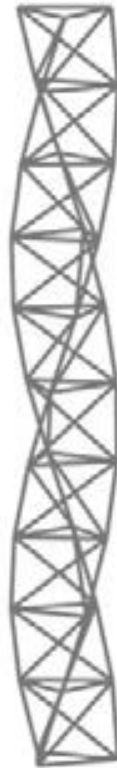

Figure 1: Nonlinear lattices with tensegrity architecture. Two-bar systems with stiffening response (a). Two-bar system with snapping response (b). Single unit (c) and double unit (d) subjected to a vertical load. Column composed of several units (e).

For the two-bar system in Fig. 1(b), the reference configuration is slightly misaligned and unstressed, with each bar inclined by an angle $\theta_{0}$. Bars are inflexible and unshearable, while remaining elastically extensible, and additional angular linearly elastic springs respond to changes in angle at nodes. Such angular springs account for the fact that the fabricated structures are monolithic, and bars nodal connections are realized as compliant hinges. If the elastic constant of rotational springs is null, this system constitutes a classic example of bistable snapping system (dotted curve in the $F$-vs- $\delta$ plot in Fig. 1,b). When the elastic constants take positive values in a certain range, the system has just one stable configuration in absence of loads, while retaining a snapping response (solid curve in the $F$-vs- $\delta$ plot in Fig. 1,b).

Figure. 1(c) shows a truss unit shaped as a T3 tensegrity system subjected to a vertical load, and the associated deformation mode, in which a relative rotation between the top and bottom bases occurs. The properties of such system depend on the initial twist angle $\varphi$ between the top and bottom bases. When $\varphi=\pi / 6$, the system behaves similarly to the two-bar system in Fig. 1(a) with null prestress [4]. When $\varphi=\pi / 6+\theta_{0}$, with $\theta_{0}<0$ a small overtwist angle, the system behaves similarly to the two-bar system in Fig. 1(b). Figure 1(d) shows a truss shaped as a double T3 system, obtained by assembling together two mirror images of the unit shown in Fig. 1(c) [14]. In this way, under a vertical force, the relative rotations between the bases of the units compensate each other, and in the resulting deformation mode, the top and bottom bases undergo just a vertical relative displacement, and only the middle base rotates. Several units can be assembled together in columns (Figure 1,e) so as to realize periodic lattices supporting the propagation of solitary or compact waves $[12,14]$. 


\section{ADDITIVE MANUFACTURING AND EXPERIMENTAL TESTING}

The geometry of the double unit is shown in Fig. 2 (left, center). The top and bottom triangular bases are inscribed in circles of radius $a=40 \mathrm{~mm}$, while $b=30 \mathrm{~mm}$ is the corresponding radius for the middle triangle. Each unit has height $h=60 \mathrm{~mm}$, and overtwist angle $\theta_{0}=-7$ degrees. Nodes were realized as spheres of diameter $d_{s}=6 \mathrm{~mm}$; the bars forming the bottom, middle, and horizontal triangles are realized with a solid circular cross section of diameter $d_{b}=4 \mathrm{~mm}$. The bars placed between the top and middle triangles, and between the middle and bottom triangle, are subdivided into three segments: the bar main body, with same cross section as mentioned above, and two terminal links to form the connections with nodal spheres. The terminal links have a solid circular cross section of diameter $d_{l}=2.2 \mathrm{~mm}$, and net length $l_{l}=4.7 \mathrm{~mm}$.
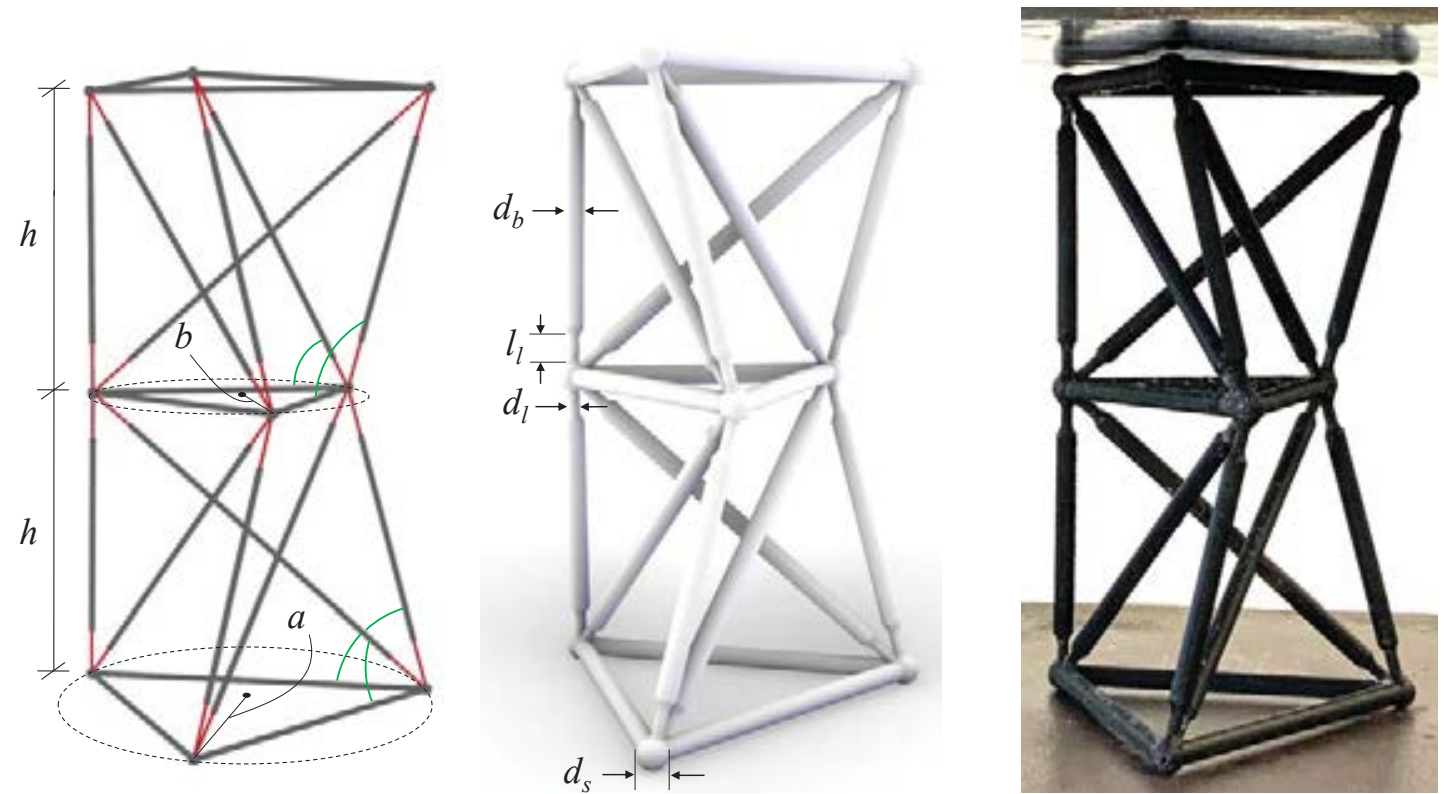

Figure 2: Geometry of a double unit and fabricated sample.

Test samples were fabricated by FDM using the Ultimaker ${ }^{\mathrm{TM}} \mathrm{S} 5$ dual extruder 3D printer, employing the Ultimaker Cura slicing software. The material chosen for the samples is polylactic acid (PLA Tough Ultimaker), while polyvinyl alcohol (PVA Ultimaker) has been used as water-soluble support material for fabrication. Figure 3(a) displays the fabrication process preview and main setup parameters in Cura, while Figure 3(b) shows one sample immediately after the fabrication process.

After removal of the supporting material, samples were subjected to a quasi-static displacementcontrol compression test. An Instron 4482 testing machine, equipped with a $10 \mathrm{kN}$ load cell, was employed to carry out the experiments, setting a testing speed of $1 \mathrm{~mm} / \mathrm{min}$. Data were collected with an acquisition frequency of $20 \mathrm{~Hz}$, while the test was recorded with a camera sampling rate of $60 \mathrm{fps}$ at $1080 \mathrm{p}$ resolution. The recorded motion of the structure and the forcevs-displacement curve depicted in Fig. 4 confirm the expected snapping behavior of the double unit. 


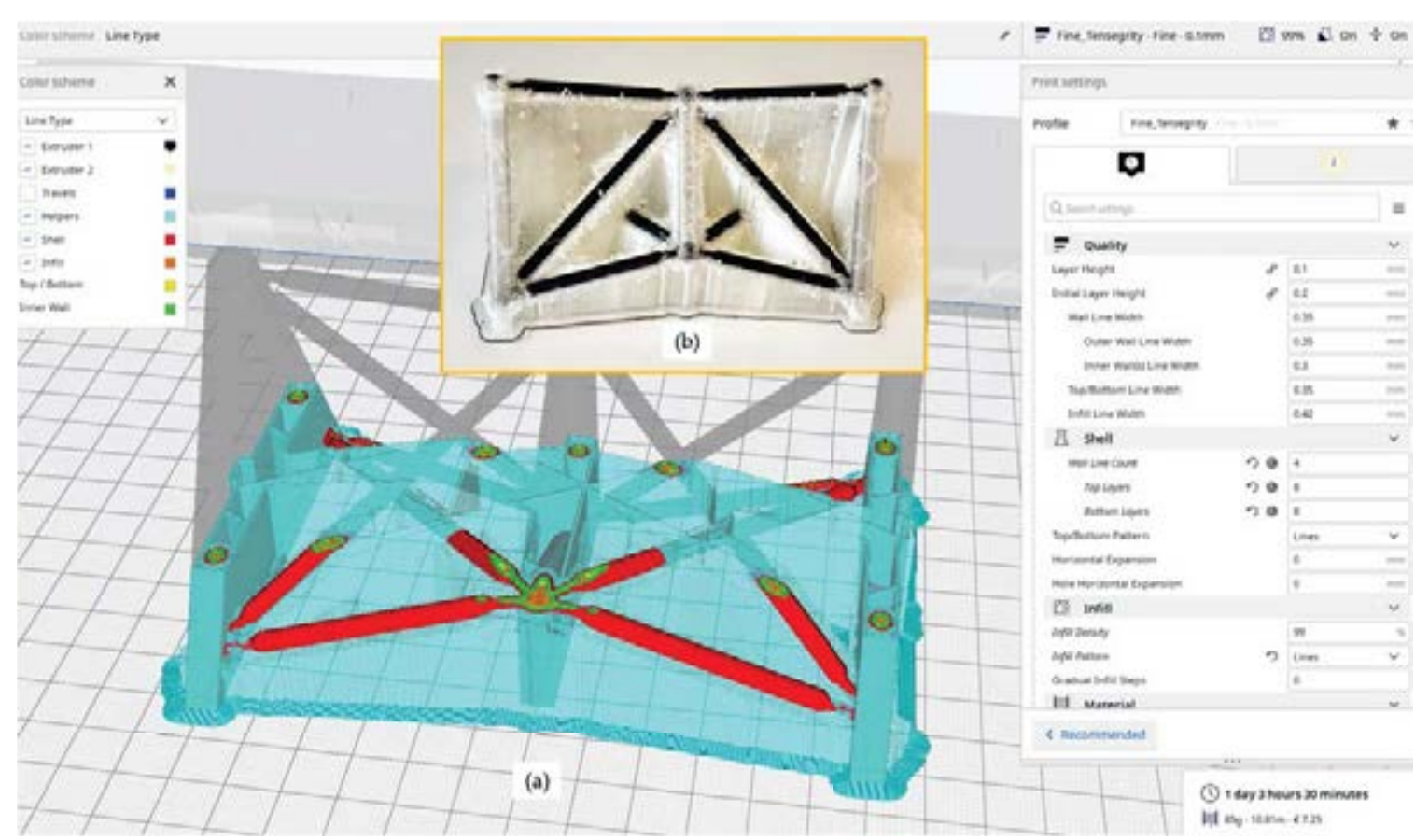

Figure 3: Fabrication preview display in Ultimaker Cura (a), and sample before removal of the supporting PVA (b).

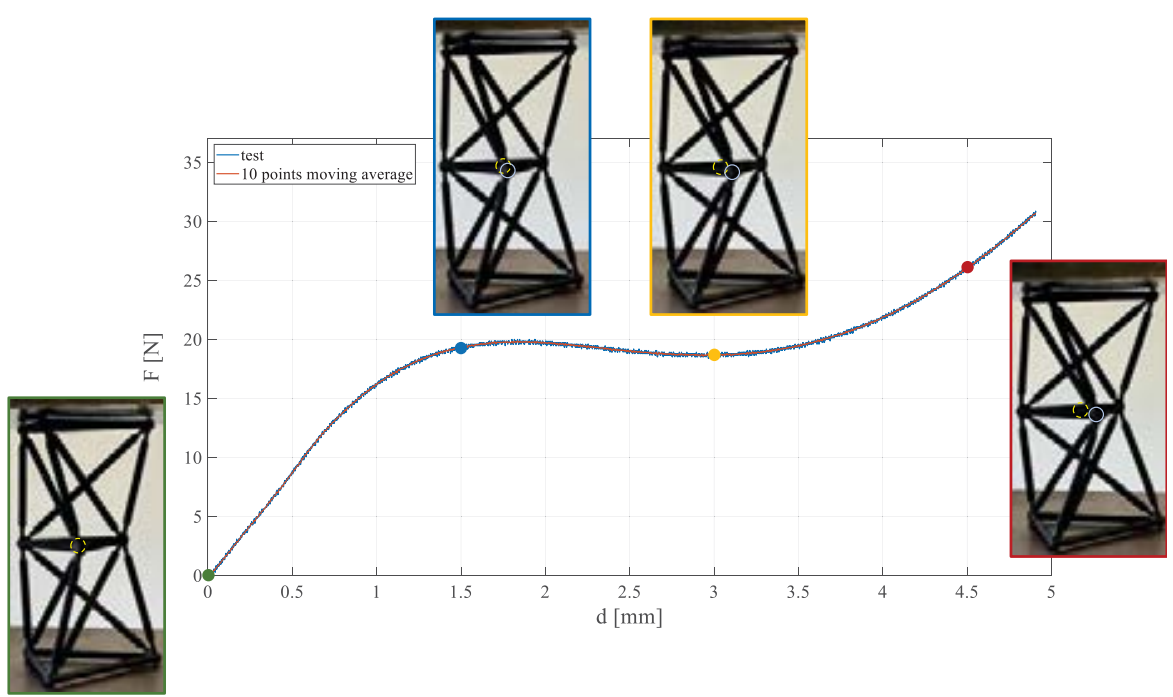

Figure 4: Compression testing results: force-vs-displacement curve.

\section{NUMERICAL SIMULATIONS}

We consider a Stick-and-Spring model $[17,18]$ in which bars are extensible but otherwise undeformable, behaving as axial springs, and equipped with angular springs responding to changes in angle between pairs of adjacent bars. Figure 2 (left) shows the location of such angular springs (green arcs, symmetric copies not shown). The axial spring constants for the bars of the horizontal triangles are computed as $E A / l_{f}$, with $E$ the Young's modulus, $A$ the cross-sectional area, and $l_{f}$ their fabrication length (inter-nodal distance). For the remaining bars, equivalent 
axial spring constants are computed considering each of them composed of three springs in series, corresponding to the three segments (two terminal links and the central bar body) they are divided in, taking into account cross-sectional area and length of each segment. Angular springs account for the bending stiffness of the terminal links, and their spring constants are computed as $E J / l_{l}$, with $J$ the inertia moment of the cross section and $l_{l}$ the link fabrication length.

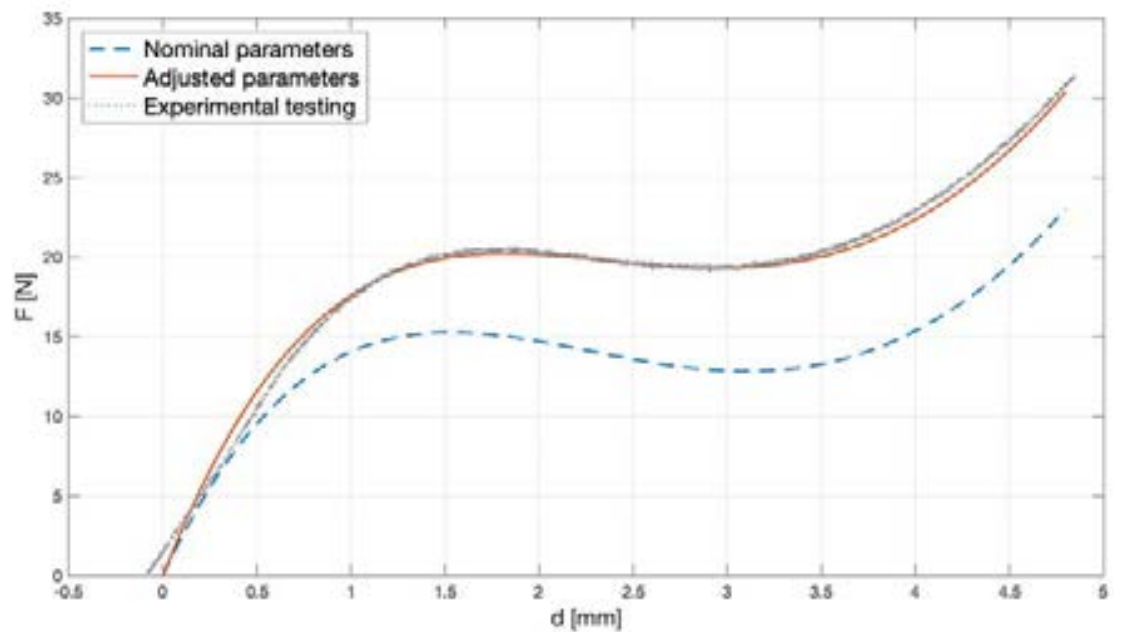

Figure 5: Test-vs-simulation results comparison.

Displacement-control numerical simulations were carried out in a regime of large displacements and rotations by imposing the vertical relative displacement between the top and bottom bases, and by computing the balancing nodal forces applied to them. In calculations, we considered the value $E=1820 \mathrm{MPa}$ for the Young's modulus, as given in the Ultimaker PLA Tough specifications. Figure 5 compares simulation results to experimental ones, and it can be observed that the simulated curve follows the experimental one qualitatively when nominal values of the geometric parameters are considered. By adjusting slightly simulation parameters, setting $\theta_{0}=-7.25$ degrees, $d_{l}=2.5 \mathrm{~mm}, l_{l}=5.0 \mathrm{~mm}$ (nominal values, $\theta_{0}=-7.00$ degrees, $d_{l}=2.2 \mathrm{~mm}, l_{l}=4.7 \mathrm{~mm}$ ), the simulated curve can be brought to be almost superposed to the numerical curve.

Impact dynamics simulations were carried out on arrays composed of several units with constant base radius $a$ (Fig. 1,e), by integrating the equations of motion written for Stick-andSpring model [18], considering nodal lumped masses $m=0.01 \mathrm{~kg}$. Figure 6 shows simulation results for an array of 20 units, in which the rightmost base is fixed, while the leftmost base is imposed a longitudinal velocity $v_{0}=2 \mathrm{~m} / \mathrm{s}$, and an angular velocity about the longitudinal axis $\omega=150 \mathrm{rad} / \mathrm{s}$, so as to trig the snapping of the leftmost unit. The nodal speed $v_{n}$ at each triangular base is represented in Fig. 6 at three time instants, showing a localized wave propagating along the array.

\section{CONCLUSIONS}

In this work, we showed that lattice metamaterials with tensegrity architecture and a highly nonlinear behavior can be effectively fabricated by simple additive manufacturing techniques, such as FDM, and that these types of nonlinear lattice units can constitute the building blocks of three-dimensional arrays with a customized mechanical response. In particular, we redesigned the double unit with snapping behavior studied in [14] by reducing bars cross section near 

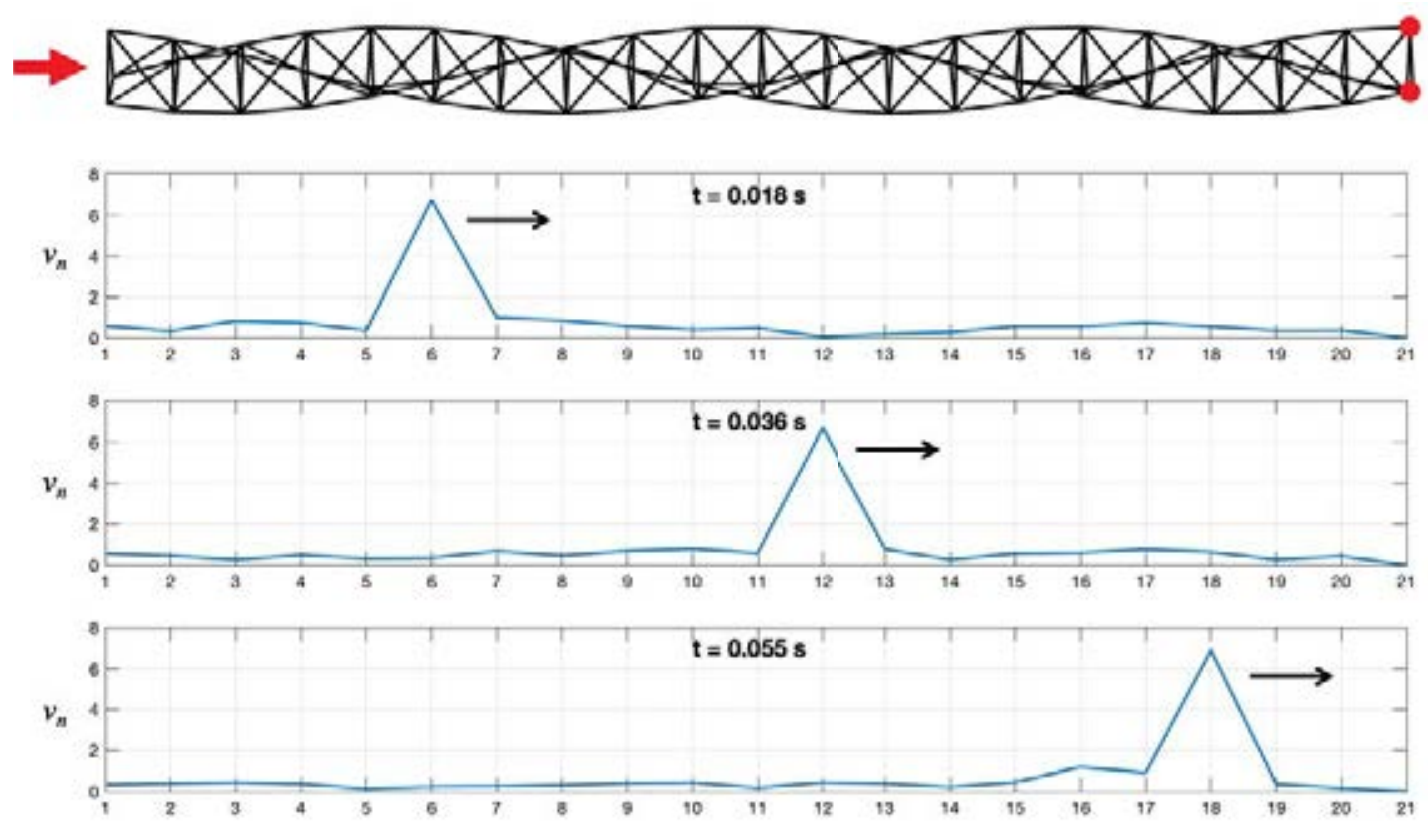

Figure 6: Impact dynamics simulation on an array of 20 units: profile of the nodal speed (m/s).

the nodes, so as to obtain an easy-to-model compliant mechanism. Fabricated samples were subjected to compression tests which confirmed the snapping behavior. The static response of the double unit was accurately simulated by calibrating a Stick-and-Spring numerical model. Such calibrated model was then employed for predicting the dynamical response of an array of several units subjected to impact loading, confirming that these type of systems support the propagation of solitary or compact waves, and that they can be realized by additive manufacturing techniques. These results can be extended to other types of tensegrity architectures and fabrication techniques to widen the range of mechanical behaviors achievable by lattice metamaterials.

\section{ACKNOWLEDGEMENTS}

A.M., C.I., N.N, E.A., and P.B. acknowledge financial support from the Italian Ministry of Education, University, and Research (MIUR) under the PRIN 2017 National Grant "3D printing: a bridge to the future" (grant number 2017L7X3CS_004).

\section{REFERENCES}

[1] Z. Liu, X. Zhang, Y. Mao, Y. Y. Zhu, Z. Yang, C. T. Chan, P. Sheng, Locally resonant sonic materials. Science, 289, 1734-1736, 2000.

[2] L.R. Meza, S. Das, J.R. Greer, Strong, lightweight, and recoverable three-dimensional ceramic nanolattices, Science, 345, 1322-1326, 2014.

[3] S. Walia, C.M. Shah, P. Gutruf, H. Nili, D.R. Chowdhury, W. Withayachumnankul, M. Bhaskaran, and S. Sriram, Flexible metasurfaces and metamaterials: A review of materials and fabrication processes at micro-and nano-scales. Applied Physics Reviews, 2(1), 011303, 2015. 
[4] I.J. Oppenheim, W.O. Williams, Geometric Effects in an Elastic Tensegrity Structure, J. Elasticity 59, 51-65, 2000.

[5] Oppenheim, I.J., Williams, W.O. Vibration of an Elastic Tensegrity Structure, Eur. J. Mech. A 20, 1023-1031, 2001.

[6] F. Fraternali, G. Carpentieri, A. Amendola, On the mechanical modeling of the extreme softening/stiffening response of axially loaded tensegrity prisms. J. Mech. Phys. Solids, 74, 136-1572014, 2015.

[7] A. Amendola, G. Carpentieri, M. De Oliveira, R.E. Skelton, F. Fraternali, Experimental investigation of the softening-stiffening response of tensegrity prisms under compressive loading. Compos. Struct. 117, 234-243, 2014.

[8] A. Micheletti, Bistable regimes in an elastic tensegrity system. Proc. R. Soc. A, 469, 2013.

[9] F. Fraternali, L. Senatore, C. Daraio, Solitary waves on tensegrity lattices. Journal of the Mechanics and Physics of Solids, 60, 1137-1144, 2012.

[10] F. Fraternali, G. Carpentieri, A. Amendola, R.E. Skelton, V.F. Nesterenko, Multiscale tunability of solitary wave dynamics in tensegrity metamaterials. Applied Physics Letters., 105, 201903, 2014.

[11] C. Davini, A. Micheletti, P. Podio-Guidugli, On the impulsive dynamics of T3 tensegrity chains, Meccanica, 51, 2763-2776, 2016.

[12] A. Micheletti, G. Ruscica, F. Fraternali, On the compact wave dynamics of tensegrity beams in multiple dimensions. Nonlinear Dynamics, 98, 2737-2753, 2019.

[13] A. Amendola, E.H. Nava, R. Goodall, I. Todd, R.E. Skelton, F. Fraternali, On the additive manufacturing and testing of tensegrity structures. Compos. Struct, 131, 66-71, 2015.

[14] Z. Vangelatos, A. Micheletti, C.P. Grigoropoulos, F. Fraternali, Design and testing of bistable lattices with tensegrity architecture and nanoscale features fabricated by multiphoton lithography. Nanomaterials, 10(4), 652, 2020.

[15] L.L. Howell, Compliant Mechanisms. In: McCarthy J. eds. $21^{\text {st }}$ Century Kinematics. Springer, 2013.

[16] M.S. Baker, L.L. Howell, On-chip actuation of an in-plane compliant bistable micromechanism. Journal of microelectromechanical systems, 11(5), 566-573, 2002.

[17] A. Favata, A. Micheletti, P. Podio-Guidugli, A Nonlinear Theory of Prestressed Elastic Stick-and-Spring Structures, International Journal of Engineering Sciences, 80, 4-20, 2014.

[18] A. Amendola, A. Favata, A. Micheletti, On the mechanical modeling of tensegrity columns subject to impact loading. Frontiers in Materials, 5, 1-22, 2018. 